\section{Estudo de base populacional dos fatores associados ao desempenho no Mini Exame do Estado Mental entre idosos: Projeto Bambuí}

\author{
A population-based study on factors associated \\ with performance on the Mini-Mental State \\ Examination in the elderly: the Bambuí Study
}

\footnotetext{
${ }_{1}$ Núcleo de Estudos em Saúde Pública e

Envelhecimento, Fundação

Oswaldo Cruz/Universidade

Federal de Minas Gerais, Belo

Horizonte, Brasil.

Correspondência

E. A. Valle

Laboratório de

Epidemiologia e Antropologia

Médica, Núcleo de Estudos

em Saúde Pública e

Envelhecimento, Fundação

Oswaldo Cruz/Universidade

Federal de Minas Gerais.

Av. Augusto de Lima 1715,

Belo Horizonte, $M G$

30190-002, Brasil.

estevaovalle@bol.com.br
}

\section{Abstract}

The aim of this study was to examine factors associated with cognitive functioning in community-dwelling older adults with low schooling. 1,588 subjects residing in Bambuí, Minas Gerais State, Brazil, and aged $>60$ years participated in this cross-sectional study. Multivariate ordinal logistic regression was used to assess associations between exploratory variables and the Mini-Mental State Examination score (MMSE): $<13$ (below the 5th percentile), 14-21 (between the 5 th percentile and the lowest quintile), and $>$ 22. Lower MMSE scores were significantly and independently associated with age $>80$ years (OR: 2.20; 95\%CI: 1.52-3.48), male gender (OR: 2.20; 95\%CI: 1.52-3.38), < 3 complete years of schooling (OR: 5.92; 95\%CI: 3.92-8.94), lack of spouse (OR: 1.91; 95\%CI: 1.39-2.62), vegetable and fruit consumption less than 5 times a week (OR: 1.94; 95\%CI: 1.39-2.62), and depressive symptoms (OR: 1.94; 95\%CI: 1.39-2.62). The results suggest that individuals with poor MMSE performance also have other markers of vulnerability.

Mental Health; Aged; Cognition Disorders
Estevão Alves Valle 1

Érico Castro-Costa 1

Josélia O. A. Firmo 1

Elizabeth Uchoa 1

Maria Fernanda Lima-Costa 1

\section{Introdução}

O Mini Exame do Estado Mental (MEEM) 1 é a escala mais utilizada para rastreamento do comprometimento cognitivo. Clinicamente, tem sido usado para detecção e acompanhamento da evolução de alterações cognitivas, bem como para o monitoramento da eficácia e efetividade de tratamentos para as mesmas. Como instrumento de pesquisa, tem sido empregado em grandes estudos populacionais $2,3,4,5$ ou acoplado a baterias de testes neuropsicológicos 6,7. Compõem o MEEM questões que se correlacionam em cinco dimensões, quais sejam: concentração, linguagem/práxis, orientação, memória e atenção, com um escore máximo de 30 pontos ${ }^{8}$. Na prática clínica, o ponto de corte 23/24 é mais comumente empregado, apresentando alta sensibilidade e especificidade para a detecção de comprometimento cognitivo e demência. Entretanto, como a performance no MEEM é influenciada pela escolaridade, outros pontos de corte têm sido recomendados no Brasil para populações com baixa escolaridade 9,10,11,12,13, mas não existe consenso quanto ao melhor ponto de coorte para esta população.

Estudos conduzidos em outros países têm descrito associações consistentes entre o baixo escore do MEEM e a idade avançada 14,15,16, a baixa escolaridade 17,18 e a presença de sintomas depressivos 19. No Brasil, associações entre o baixo escore do MEEM, baixa escolaridade e a idade 
avançada já foram descritas 9,10,11,12,13, mas a sua associação com outros fatores ainda não foi investigada em estudos de base populacional.

O presente trabalho foi realizado entre participantes da linha de base da coorte de idosos de Bambuí ${ }^{20}$, com o objetivo de examinar fatores associados ao desempenho no teste do MEEM em uma população idosa com baixa escolaridade.

\section{Metodologia}

O Projeto Bambuí é um estudo de coorte de base populacional sobre saúde de idosos, desenvolvido na cidade de mesmo nome, situada em Minas Gerais, Brasil. A linha de base da coorte foi constituída em 1997. Os participantes da pesquisa foram identificados por meio de um censo completo realizado no município pela equipe do projeto. Maiores detalhes podem ser vistos em publicação anterior 20. Todos os moradores com sessenta anos ou mais de idade em lo de janeiro de 1997 ( $n=1.742$ ) foram convidados a participar do estudo e 1.606 (92,2\%) participaram da linha de base da coorte. Para o presente trabalho foram selecionados todos os participantes que responderam ao questionário MEEM.

A variável dependente deste estudo é o escore do MEEM, obtido por meio de uma versão do instrumento traduzida para o português, com algumas adaptações ${ }^{21}$. Na ausência de um ponto de corte consensual para populações com baixa escolaridade, foi considerada a distribuição em percentis dos escores do MEEM na população estudada, como previamente sugerido para esta população ${ }^{13}$. O MEEM foi aplicado por entrevistadores com pelo menos 11 anos completos de escolaridade, selecionados entre moradores da cidade de Bambuí. O treinamento foi realizado por psiquiatra qualificada (E. U.) e os entrevistadores foram certificados somente após avaliação da confiabilidade intra e entre observadores. Maiores detalhes podem ser vistos em publicação anterior 20 .

As variáveis independentes do estudo incluíram características sócio-demográficas, estilos de vida relacionados à saúde, indicadores das condições de saúde e usos de serviços de saúde. Entre as características sócio-demográficas foram consideradas idade, sexo, número de anos completos de escolaridade e situação conjugal (ausência de cônjuge foi definida pela condição de solteiro, divorciado ou separado e viúvo). Entre os estilos de vida foram incluídos tabagismo (considerando-se como fumante atual aquele que já fumou pelo menos 100 cigarros ao longo da vida e ainda fumava, e como ex-fumante aquele que havia parado de fumar) 22 e consumo semanal de frutas e hortaliças (cinco ou mais vezes por semana nos últimos 12 meses ou menos).

Os indicadores de condições de saúde considerados neste trabalho foram sintomas depressivos, diabetes mellitus e hipertensão arterial. A presença de sintomas depressivos foi avaliada por meio do General Health Questionnaire (GHQ-12), utilizando-se ponto de corte (4/5), previamente validado na população em estudo 23. Hipertensão arterial sistêmica foi definida segundo os critérios estabelecidos pelo VII JNC Report 24 , ou seja, média da pressão sistólica $\geq 140 \mathrm{mmHg}$ e/ou média da pressão diastólica $\geq 90 \mathrm{mmHg}$ ou tratamento atual para hipertensão. Foram realizadas três medidas de pressão arterial, com intervalos de 2 minutos entre elas, após um descanso inicial de 5 minutos e depois de 30 minutos sem ingestão de álcool ou cafeína, considerando-se a média da segunda e a terceira medida e descartando-se a primeira. A definição de diabetes foi baseada no nível de glicose em jejum, considerando-se valores iguais ou superiores a $126 \mathrm{mg} / \mathrm{dL}$ e/ou uso atual de insulina ou hipoglicemiante oral, de acordo com os critérios da American Diabetes Association 25. Entre os indicadores de usos de serviços de saúde foram considerados tempo decorrido após a última visita a um médico e número de hospitalizações nos últimos 12 meses.

O Projeto Bambuí foi aprovado na Comissão de Ética da Fundação Oswaldo Cruz. Todos os participantes assinaram o termo de consentimento pós-informado.

\section{Análise estatística}

A análise estatística foi baseada em odds ratio (OR) estimados por meio da regressão logística ordinal univariada e multivariada; a proporcionalidade do OR em cada modelo foi examinada usando-se o teste de Wald. Todas as variáveis consideradas no presente trabalho foram incluídas uma a uma no modelo multivariado, que incluiu inicialmente as características sócio-demográficas, seguidas pelos hábitos de vida, pelos indicadores das condições de saúde e pelos indicadores de usos de serviços de saúde. Foram mantidas no modelo multivariado final aquelas que permaneceram significativamente associadas $(\mathrm{p}<0,05)$ ao escore do MEEM. As análises foram realizadas utilizando-se o Stata Software Package, versão 10.0 (Stata Corp., College Station, Estados Unidos). 


\section{Resultados}

Participaram do presente trabalho 1.558 indivíduos, que correspondem a $97 \%$ dos participantes da linha de base da coorte. A média de idade foi de 69 anos (desvio-padrão = 7,3), predominando o sexo feminino $(60,1 \%)$ e a baixa escolaridade (64,8\% possuíam escolaridade inferior a quatro anos completos). Outras características dos participantes do estudo podem ser vistas na Tabela 1 .

A mediana do escore do MMSE entre os participantes do estudo foi igual a 26. Escores iguais ou inferiores a $13(4,3 \%)$ corresponderam aos valores abaixo do 5o percentil, entre 14 e 21 (17,7\%) a valores entre o 5o, e abaixo do 25 o percentil e escores $\geq 22$ (78\%) corresponderam aos quartis superiores.

Na Tabela 2 estão apresentados os resultados da análise univariada da associação entre escore do MEEM, características sócio-demográficas e estilos de vida. OR estatisticamente significantes foram observadas para faixas etária mais velhas (70-79 e 80 ou mais anos), sexo masculino, escolaridade mais baixa (inferior a quatro anos), ausência de cônjuge, tabagismo atual e passado, e ingestão menos freqüente de frutas e hortaliças (menos de cinco vezes por semana).

Entre os indicadores das condições de saúde e de uso de serviços de saúde, presença de sintomas depressivos e número de hospitalizações nos últimos 12 meses (uma e duas ou mais) apresentaram associações significantes com escores mais baixos do MEEM na análise univariada (Tabela 3).

Os resultados finais da análise multivariada dos fatores associados aos escores do MEEM es- tão apresentados na Tabela 4. Associações independentes com escores mais baixos do MEEM foram observadas para faixa etária mais velha $(\geq$ 80 anos), sexo masculino, escolaridade inferior a quatro anos, ausência de cônjuge, consumo de frutas e hortaliças menos de cinco vezes por semana e presença de sintomas depressivos.

\section{Discussão}

Os resultados deste trabalho mostraram a existência de associações entre baixos escores do MEEM e idade, sexo, escolaridade, situação conjugal, consumo de frutas e hortaliças e presença de sintomas depressivos. Essas associações persistiram mesmo após ajustamentos entre elas e foram independentes de outras variáveis consideradas no estudo.

A idade é um dos determinantes mais importantes do declínio cognitivo. Estudos de base populacional têm mostrado de forma consistente que existe uma piora do desempenho no MEEM com o aumento da idade $2,3,4,5,6,14,17,18$. Os resultados deste trabalho estão de acordo com essas observações.

Com referência ao sexo, estudos epidemiológicos têm mostrado resultados discordantes quanto à sua associação com a função cognitiva em idosos. Em San Antonio, Estados Unidos, observou-se que o sexo não estava associado ao desempenho cognitivo 2. Estudos epidemiológicos conduzidos no Reino Unido ${ }^{3}$ e na Coréia do Sul 5 descreveram pior desempenho cognitivo entre as mulheres; já na Austrália, observou-se que os homens apresentavam pior função cognitiva 14 .

Tabela 1

Características dos 1.558 participantes do estudo. Projeto Bambuí, Minas Gerais، Brasil. 
Análise univariada da associação entre escore do Mini Exame do Estado Mental (MEEM), características sócio-demográficas e hábitos relacionados à saúde. Projeto Bambuí, Minas Gerais, Brasil.

\begin{tabular}{|c|c|c|c|c|}
\hline \multirow[t]{2}{*}{ Características } & \multicolumn{2}{|c|}{ Escore do MEEM (n) } & \multirow[b]{2}{*}{$\begin{array}{c}\leq 13 \\
\text { (abaixo do 5o } \\
\text { percentil) }\end{array}$} & \multirow[t]{2}{*}{ OR (IC95\%) } \\
\hline & $\geq 22$ & $\begin{array}{l}14-21 \\
\text { (5o percentil e } \\
\text { quintil inferior) }\end{array}$ & & \\
\hline \multicolumn{5}{|l|}{ Faixa etária (anos) } \\
\hline $60-69$ & 707 & 110 & 20 & 1,00 \\
\hline $70-79$ & 329 & 70 & 17 & $1,44(1,10-1,88)$ \\
\hline$\geq 80$ & 91 & 32 & 23 & $3,33(2,36-4,71)$ \\
\hline \multicolumn{5}{|l|}{ Sexo } \\
\hline Feminino & 671 & 95 & 23 & 1,00 \\
\hline Masculino & 456 & 117 & 37 & $1,74(2,36-4,71)$ \\
\hline \multicolumn{5}{|l|}{ Escolaridade (anos completos) } \\
\hline$\geq 4$ & 508 & 19 & 4 & 1,00 \\
\hline$<4$ & 619 & 193 & 55 & $6,94(4,76-10,11)$ \\
\hline \multicolumn{5}{|l|}{ Situação conjugal } \\
\hline Presença de cônjuge & 631 & 118 & 20 & 1,00 \\
\hline Ausência de cônjuge & 584 & 158 & 47 & $1,64(1,28-2,08)$ \\
\hline \multicolumn{5}{|l|}{ Tabagismo } \\
\hline Nunca fumou & 720 & 100 & 28 & 1,00 \\
\hline Ex-fumante & 223 & 42 & 16 & $1,44(1,06-1,96)$ \\
\hline Fumante atual & 184 & 70 & 16 & $2,03(1,51-2,72)$ \\
\hline \multicolumn{5}{|c|}{ Consumo de legumes, frutas ou verduras frescas } \\
\hline 5 ou mais vezes por semana & 764 & 106 & 28 & 1,00 \\
\hline Menos de 5 vezes por semana & 451 & 170 & 39 & $2,60(2,04-3,32)$ \\
\hline
\end{tabular}

OR (IC95\%): odds ratio não ajustado e intervalo de 95\% de confiança, estimado por meio de regressão logística ordinal (os valores de p referentes ao teste de Wald para linhas paralelas variaram entre 0,118 e 0,948, indicando que todos os modelos não violaram a premissa de proporcionalidade do OR).

No Brasil, na cidade de Catanduva, em um estudo transversal observou-se pior desempenho cognitivo entre as mulheres 4 . No entanto, após ajustamentos por variáveis de confusão, verificou-se nessa mesma cidade que a incidência de déficit cognitivo era semelhante entre homens e mulheres 26 . No presente estudo transversal, os homens apresentaram piores escores no MEEM, constatando-se que esta associação persistia após ajustamentos por outras características relevantes. Essas observações sugerem que características particulares de cada população podem ter influência na associação entre função cognitiva e sexo.

A escolaridade é um dos fatores mais consistentemente descritos como associados ao desempenho no MEEM 2,3,4,5,6,14,17,18. Os resultados deste trabalho são consistentes com esses achados, uma vez que foi encontrada forte associação entre pior escolaridade e escores mais baixos do MEEM. Nossos resultados acrescentam em relação aos trabalhos anteriores por mostrar que, mesmo em uma população na qual predominam níveis muito baixos de escolaridade, a influência da escolaridadeno escore do MEEM está presente.

A ausência de cônjuge foi outra característica fortemente associada ao pior desempenho no MEEM entre idosos bambuienses. Em alguns estudos observou-se que indivíduos solteiros, sobretudo aqueles que moram sozinhos, estão sob maior risco de deterioração cognitiva 27 e desenvolvimento de demência 28,29. A associação entre ausência de cônjuge e maior risco para quadros demenciais também tem sido descrita 29.

A relação entre o tabagismo e a cognição é controversa. Resultados de um estudo de casocontrole sugeriram haver um papel neuroprotetor da nicotina 30 , ao passo que em um estudo de coorte observou-se que tabagistas apresentam maior decréscimo do escore de MEEM ao longo do tempo, em comparação aos não-fumantes 31 . Uma meta-análise recente, contemplando uma ampla variação de desfechos relacionados à cognição, mostrou que os tabagistas têm riscos $40 \%$ a $80 \%$ maiores de piora da cognição com relação aos idosos que não fumam 32 . No presente traba- 
Análise univariada da associação entre escore do Mini Exame do Estado Mental (MEEM), indicadores de condições de saúde e de usos de serviços de saúde. Projeto Bambuí, Minas Gerais, Brasil.

\begin{tabular}{|c|c|c|c|c|}
\hline \multirow[t]{4}{*}{ Características } & \multicolumn{3}{|c|}{ Escore do MEEM (n) } & \multirow[t]{4}{*}{ OR (IC95\%) } \\
\hline & $\geq 22$ & $14-21$ & $\leq 13$ & \\
\hline & & (5ㅇ percentil e & (abaixo do 5o & \\
\hline & & quintil inferior) & percentil) & \\
\hline \multicolumn{5}{|l|}{ Faixa etária (anos) } \\
\hline $60-69$ & 707 & 110 & 20 & 1,00 \\
\hline $70-79$ & 329 & 70 & 17 & $1,44(1,10-1,88)$ \\
\hline$\geq 80$ & 91 & 32 & 23 & $3,33(2,36-4,71)$ \\
\hline \multicolumn{5}{|l|}{ Sexo } \\
\hline Feminino & 671 & 95 & 23 & 1,00 \\
\hline Masculino & 456 & 117 & 37 & $1,74(2,36-4,71)$ \\
\hline \multicolumn{5}{|l|}{ Escolaridade (anos completos) } \\
\hline$\geq 4$ & 508 & 19 & 4 & 1,00 \\
\hline$<4$ & 619 & 193 & 55 & $6,94(4,76-10,11)$ \\
\hline \multicolumn{5}{|l|}{ Situação conjugal } \\
\hline Presença de cônjuge & 631 & 118 & 20 & 1,00 \\
\hline Ausência de cônjuge & 584 & 158 & 47 & $1,64(1,28-2,08)$ \\
\hline \multicolumn{5}{|l|}{ Tabagismo } \\
\hline Nunca fumou & 720 & 100 & 28 & 1,00 \\
\hline Ex-fumante & 223 & 42 & 16 & $1,44(1,06-1,96)$ \\
\hline Fumante atual & 184 & 70 & 16 & $2,03(1,51-2,72)$ \\
\hline \multicolumn{5}{|c|}{ Consumo de legumes, frutas ou verduras frescas } \\
\hline 5 ou mais vezes por semana & 764 & 106 & 28 & 1,00 \\
\hline Menos de 5 vezes por semana & 451 & 170 & 39 & $2,60(2,04-3,32)$ \\
\hline
\end{tabular}

GHQ: General Health Questionnaire.

OR (IC95\%): odds ratio não ajustado e intervalo de 95\% de confiança, estimado por meio de regressão logística ordinal (os valores de p referentes ao teste de Wald para linhas paralelas variaram entre 0,074 e 0,559, indicando que todos os modelos não violaram a premissa de proporcionalidade do OR).

lho, observou-se maior prevalência de fumantes atuais e passados entre idosos com escores mais baixos do MEEM, mas esta associação desapareceu após ajustamentos por variáveis de confusão.

Uma observação que merece ser destacada foi a menor ingestão de frutas e hortaliças entre idosos com pior desempenho no MEEM. A ingestão insuficiente desses alimentos está relacionada ao pior prognóstico cognitivo. Há estudos que demonstram haver relação entre o consumo de frutas e hortaliças e a preservação da cognição 33,34 . A hipótese mais aceita é que altos teores de antioxidantes, como as vitaminas E e C, além de substâncias bioativas, como carotenóides e flavonóides, contidos nesses alimentos atenuam o estresse oxidativo e os processos neurodegerativos relacionados ao declínio cognitivo e às demências. Além disso, por serem fontes de vitaminas B12, B6 e ácido fólico, frutas e hortaliças poderiam ter papel benéfico à cognição 35 . Devido à natureza transversal do presente trabalho, não é possível saber se a ingestão menos freqüente de frutas e hortaliças reflete um hábito ao longo da vida que contribuiu para a piora da cognição, ou se é uma conseqüência da mesma, refletindo mudanças na preferência e seleção de alimentos, não incomuns nos processos demenciais. Uma outra observação consistente em diversas populações é a associação entre sintomas depressivos e comprometimento cognitivo em idosos 36,37 . No presente trabalho observou-se uma associação independente e graduada com escores inferiores do MEEM. A natureza transversal do estudo não permite saber se esses sintomas são um fator de risco ou se são conseqüência da deterioração cognitiva 20,21. Estudos longitudinais são necessários para um melhor entendimento dessa associação na população estudada.

Não se observou associação significante entre hipertensão arterial, diabetes mellitus e escore do MEEM na população estudada, confirmando observações em outras populações 14,38. Entre idosos bambuienses também não se constatou 
Resultados finais da análise multivariada dos fatores associados ao escore do Mini Exame do Estado Mental (MEEM). Projeto Bambuí, Minas Gerais, Brasil.

\begin{tabular}{|c|c|}
\hline Características & OR (IC95\%) \\
\hline \multicolumn{2}{|l|}{ Faixa etária (anos) } \\
\hline $60-69$ & 1 \\
\hline $70-79$ & $1,18(0,87-1,61)$ \\
\hline$\geq 80$ & $2,30(1,52-3,48)$ \\
\hline \multicolumn{2}{|l|}{ Sexo } \\
\hline Feminino & 1 \\
\hline Masculino & $2,20(1,59-3,04)$ \\
\hline \multicolumn{2}{|c|}{ Escolaridade (anos completos) } \\
\hline$\geq 4$ & 1 \\
\hline$<4$ & $5,91(3,92-8,94)$ \\
\hline \multicolumn{2}{|l|}{ Situação conjugal } \\
\hline Presença de cônjuge & 1 \\
\hline Ausência de cônjuge & $1,91(1,39-2,63)$ \\
\hline \multicolumn{2}{|c|}{ Consumo de legumes, frutas ou verduras frescas (por semana) } \\
\hline 5 ou mais vezes & 1 \\
\hline Menos de 5 vezes & $1,94(1,46-2,59)$ \\
\hline \multicolumn{2}{|c|}{ Sintomas depressivos (escore do GHQ-12 4/5) } \\
\hline Não & 1 \\
\hline Sim & $1,94(1,46-2,56)$ \\
\hline
\end{tabular}

GHQ: General Health Questionnaire.

OR (IC95\%): odds ratio não ajustado e intervalo de 95\% de confiança, estimado por meio de regressão logística ordinal por todas as variáveis listadas na tabela (os valores de $p$ referentes ao teste de Wald para linhas paralelas $=0,320$, indicando que o modelo multivariado final não violou a premissa de proporcionalidade do OR).

associação entre escore do MEEM e uso de serviços de saúde, tanto no que se refere ao tempo decorrido após a última consulta médica quanto ao número de hospitalizações. Uma associação entre pior escore do MEEM e ocorrência de hospitalizações foi observada na análise não ajustada, mas esta associação desapareceu após ajustamentos por fatores de confusão.

Com relação a aspectos metodológicos deste trabalho, é importante ressaltar que existem diferentes opções para análise dos escores obtidos por meio do MEEM em estudos epidemiológicos. A opção predominante é a adoção de um ponto de corte previamente validado em indivíduos com escolaridade mais alta (geralmente $23 / 24$ ), assumindo-se que pessoas com escores abaixo deste valor apresentam alta probabilidade de déficit cognitivo ${ }^{1}$. Entretanto, a adoção desse ponto de coorte para indivíduos com baixa escolaridade é questionável. No Brasil, têm sido propostos pontos de corte mais baixos para pessoas sem escolaridade formal ou com níveis muito baixos de escolaridade. Os escores propostos são bastante díspares, com valores variando entre 12/13 e 18/19 9,10,11,12. Quando aplicados a uma mesma população, os pontos de coorte propostos apresentam concordância muito baixa, refletindo a ausência de consenso sobre o tema ${ }^{13}$. Na ausência de um ponto de coorte consistente, uma alternativa é considerar os escores do MEEM como variáveis contínuas e fazer comparações entre as médias correspondentes; esta estratégia é adequada quando os escores apresentam distribuição normal. Entretanto, esse não é o caso da distribuição dos escores do MEEM na população de base da coorte de Bambuí. Nessa população, os escores do MEEM não apresentavam distribuição normal e não era possível normalizar esta distribuição por meio de transformações habituais ${ }^{13}$. Dessa forma, no presente trabalho considerou-se a distribuição em percentis do escore do MEEM. Essa opção tem sido recomendada para estudos de base populacional, assumindo-se que valores abaixo do 5 o percentil identificam indivíduos com alta probabilidade de déficit cognitivo, e valores situados no quartil inferior identificam casos suspeitos 13,39. Essa estratégia é vantajosa porque, além de possibilitar a investigação de associações entre o escore do MEEM e as características 
de interesse, permite também verificar se existe um gradiente nesta associação.

Em resumo, nossos resultados mostraram que os fatores associados ao desempenho no MEEM na população estudada foram semelhantes àqueles descritos em populações com escolaridade mais alta. A associação entre menor ingestão de frutas e hortaliças e pior desempenho no MEEM observada neste trabalho é preocupante e aponta para a necessidade de intervenções visando ao

\section{Resumo}

O objetivo deste trabalho foi examinar os fatores associados ao desempenho no Mini Exame do Estado Mental (MEEM) entre idosos com baixa escolaridade. Participaram deste estudo transversal 1.558 indivíduos com $\geq 60$ anos residentes na cidade de Bambuí, Minas Gerais, Brasil. Foi utilizada a regressão logística ordinal para investigar as associações existentes entre escores do MEEM e características selecionadas. Os escores foram estratificados em percentis: abaixo do 5 o ( $\leq 13)$, entre o 5 o e abaixo do 25o (14-21) e quartis superiores $(\geq 22)$. Associações positivas e independentes com o pior desempenho no MEEM foram observadas para faixa etária $\geq 80$ anos $(O R=2,20$; IC95\%: 1,52 $3,48)$, sexo masculino $(O R=2,20$; IC95\%: 1,52-3,38), escolaridade $<4$ anos $(O R=5,92 ;$ IC95\%: 3,92-8,94), não possuir cônjuge (OR = 1,91; IC95\%: 1,39-2,62), consumo de vegetais $<5$ vezes por semana $(O R=1,94$; IC95\%: 1,39-2,62) e sintomas depressivos $(O R=1,94$; IC95\%: 1,46-2,56). Os resultados indicam que os idosos com pior desempenho no MEEM eram aqueles que possuíam outros marcadores de vulnerabilidade. aumento da ingestão destes alimentos, que pode ser benéfica tanto para as condições gerais de saúde quanto para a evolução das funções cognitivas. Os resultados do presente trabalho mostraram que idosos com pior desempenho no MEEM são aqueles que possuem também outros marcadores de vulnerabilidade. Políticas voltadas para idosos com comprometimento cognitivo devem considerar essa vulnerabilidade, adotando-se medidas que visem à sua compensação.

\section{Colaboradores}

E. A. Valle participou no delineamento do estudo, análise dos dados e redação do trabalho. E. Castro-Costa participou da análise dos dados e da revisão crítica do manuscrito. J. O. A. Firmo e E. Uchoa participaram do delineamento do estudo e revisão crítica do manuscrito. M. F. Lima-Costa foi responsável pela supervisão da análise dos dados, redação do trabalho e revisão final do artigo.

\section{Agradecimentos}

Este trabalho foi custeado pela Financiadora de Estudos e Projetos (FINEP) e pela Fundação de Amparo à Pesquisa do Estado de Minas Gerais (FAPEMIG). E. Uchoa e M. F. Lima-Costa possuem bolsas de produtividade em pesquisa do Conselho Nacional de Desenvolvimento Científico e Tecnológico (CNPq) e E. C. Costa possui bolsa de pós-doutorado da FAPEMIG. 


\section{Referências}

1. Folstein MF, Folstein SE, McHugh PR. Mini-Mental State: a practical method for grading the cognitive state of patients for the clinician. J Psychiat Res 1975; 12:189-98.

2. Espino DV, Lichtenstein MJ, Palmer RF, Hazuda HP. Ethnic differences in mini-mental state examination (MMSE) scores: where you live makes a difference. J Am Geriatr Soc 2001; 49:538-48.

3. Huppert FA, Cabelli ST, Matthews FE. MRC Cognitive Function and Ageing Study. Brief cognitive assessment in a UK population sample - distributional properties and the relationship between the MMSE and an extended mental state examination. BMC Geriatr 2005; 5:7.

4. Herrera Jr. E, Caramelli P, Silveira AS, Nitrini R. Epidemiologic survey of dementia in a community-dwelling Brazilian population. Alzheimer Dis Assoc Disord 2002; 16:103-8.

5. Han C, Jo SA, Jo I, Kim E, Park MH, Kang Y. An adaptation of the Korean mini-mental state examination (K-MMSE) in elderly Koreans: demographic influence and population-based norms (the AGE study). Arch Gerontol Geriatr 2007; 47:302-10.

6. Morris JC, Heyman A, Mohs RC, Hughes JP, van Belle G, Fillenbaum G, et al. The Consortium to Establish a Registry for Alzheimer's Disease (CERAD). Part I: clinical and neuropsychological assessment for Alzheimer's disease. Neurology 1989; 39: 1159-65.

7. Roth M, Tym E, Mountjoy CQ, Huppert FA, Hendrie $\mathrm{H}$, Verma S, et al. CAMDEX: a standardized instrument for the diagnosis of mental disorder in the elderly with special reference to the early detection of dementia. Br J Psychiatry 1986; 149:698-709.

8. Jones R, Gallo J. Dimensions of the Mini-Mental State Examination among community dwelling older adults. Psychol Med 2000; 30:605-18.

9. Bertolucci PHF, Brucki SMD, Campacci S, Juliano Y. O Mini-Exame do Estado Mental em uma população geral. Impacto da escolaridade. Arq Neuropsiquiatr 1994; 52:1-7.

10. Almeida OP. Mini Exame do Estado Mental e o diagnóstico de demência no Brasil. Arq Neuropsiquiatr 1998; 56:605-12.

11. Caramelli P, Herrera Jr. E, Nitrini R. O Mini Exame do Estado Mental no diagnóstico de demência em idosos analfabetos. Arq Neuropsiq 1999; 57 Suppl 11:7.

12. Lourenço RA, Veras RP. Mini exame do estado mental: características psicométricas em idosos ambulatoriais. Rev Saúde Pública 2006; 40:712-9.

13. Castro-Costa E, Fuzikawa C, Uchoa E, Firmo JOA, Lima-Costa MF. Norms for the Mini-Mental State Examination: adjustment of the cut-off point in population-based studies (evidences from the Bambuí Health Aging study). Arq Neuropsiq 2008; 66:524-8.

14. Anderson TM, Brodaty H, Trollor JN, Andrews G. Effects of sociodemographic and health variables on Mini-Mental State Exam scores in older Australians. Am J Geriatr Psychiatry 2007; 15:467-76.
15. Laks J, Contino ALB, Paula EO, Engelhardt E. MiniMental State Examination norms in a communitydwelling sample of elderly with low schooling in Brazil. Cad Saúde Pública 2007; 23:315-9.

16. Dufouil C, Clayton D, Brayne C, Chi LY, Dening TR, Paykel ES, et al. Population norms for the MMSE in the very old: estimates based on longitudinal data. Mini-Mental State Examination. Neurology 2000; 55:1609-13.

17. Crum RM, Anthony JC, Bassett SS, Folstein MF Population-based norms for the Mini-Mental State Examination by age and education level. JAMA 1993; 269:2386-91.

18. Butler SM, Ashford JW, Snowdon DA. Age, education, and changes in the Mini-Mental State Exam scores of older women: findings from the Nun Study. J Am Geriatr Soc 1996; 44:675-81.

19. Ganguli M, Du Y, Dodge HH, Ratcliff GG, Chang CC. Depressive symptoms and cognitive decline in late life. A prospective epidemiological study. Arch Gen Psychiatry 2006; 63:153-60.

20. Lima-Costa MFF, Uchoa E, Guerra HL, Firmo JOA, Vidigal PG, Barreto SM. The Bambuí health and ageing study (BHAS): methodological approach and preliminary results of a population-based cohort study of the elderly in Brazil. Rev Saúde Pública 2000; 34:126-35.

21. Seabra MLV, Concílio GV, Villares JB, Carlini EA. Avalição do teste "Mini-mental state" em voluntários e pacientes brasileiros. Rev ABP-APL 1990; 12:1-7.

22. National Center for Health Statistics, Centers for Disease Control and Prevention. NCHS definitions: cigarrete smoking. http://www.cdc.gov/nchs/ datawh/nchsdefs/cigarettesmoking.htm (acessado em 06/Jun/2007).

23. Costa E, Barreto SM, Uchoa E, Firmo JO, LimaCosta MF, Prince M. Is the GDS-30 better than the GHQ-12 for screening depression in elderly people in the community? The Bambuí Health Aging Study (BHAS). Int Psychogeriatr 2006; 18:493-503.

24. Chobanian AV, Bakris GL, Black HR, Cushman WC, Green LA, Izzo JL, et al. The seventh report of the joint national committee on prevention, detection, evaluation, and treatment of high blood pressure: the JNC 7 report. JAMA 2003; 289:2560-72.

25. The Expert Committee on the Diagnosis and Classification of Diabetes Mellitus: follow-up report on the diagnosis of diabetes mellitus. Diabetes Care 2003; 26:3160-7.

26. Nitrini R, Caramelli P, Herrera Jr. E, Bahia VS, Caixeta LF, Radanovic M, et al. Incidence of dementia in a community-dwelling Brazilian population. Alzheimer Dis Assoc Disord 2004; 18:241-6.

27. Bassuk SS, Glass TA, Berkman LF. Social disengagement and incident cognitive decline in community-dwelling elderly persons. Ann Intern Med 1999; 131:165-73.

28. Fratiglioni L, Wang HX, Ericsson K, Maytan M, Winblad B. Influence of social network on occurrence of dementia: a community-based longitudinal study. Lancet 2000; 355:1315-9. 
29. Helmer C, Damon D, Letenneur L, Fabrigoule C, Barberger-Gateau P, Lafont S, et al. Marital status and risk of Alzheimer's disease: a French population-based cohort study. Neurology 1999; 53: 1953-8.

30. Almeida OP, Hulse GK, Lawrence D, Flicker L. Smoking as a risk factor for Alzheimer's disease: contrasting evidence from a systematic review of case-control and cohort studies. Addiction 2002; 97:15-28.

31. Ott A, Andersen K, Dewey ME, Letenneur L, Brayne C, Copeland JR, et al. EURODEM Incidence Research Group. Effect of smoking on global cognitive function in nondemented elderly. Neurology 2004; 62:920-4.

32. Anstey KJ, von Sanden C, Salim A, O'Kearney R. Smoking as a risk factor for dementia and cognitive decline: a meta-analysis of prospective studies. Am J Epidemiol 2007; 166:367-78.

33. Morris MC, Evans DA, Tangney CC, Bienias JL, Wilson RS. Associations of vegetable and fruit consumption with age-related cognitive change. Neurology 2006; 67:1370-6.

34. Kang JH, Ascherio A, Grodstein F. Fruit and vegetable consumption and cognitive decline in aging women. Ann Neurol 2005; 57:713-20.
35. Donini LM, De Felice MR, Cannella C. Nutritional status determinants and cognition in the elderly. Arch Gerontol Geriatr 2007; 44 Suppl 1:143-53.

36. van Ojen R, Hooijer C, Bezemer D, Jonker C, Lindeboom J, van Tilburg W. Late-life depressive disorder in the community, I: the relationship between MMSE score and depression in subjects with and without psychiatric history. Br J Psychiatry 1995; 166:311-9.

37. Chan SS, Lam LC, Tam CW, Lui VW, Chan WC, Wong S, et al. Prevalence of clinically significant depressive symptoms in an epidemiologic sample of community-dwelling elders with milder forms of cognitive impairment in Hong Kong SAR. Int J Geriatr Psychiatry 2007; 22:28.

38. Fischer P, Zehetmayer S, Bauer K, Huber K, Jungwirth S, Tragl KH. Relation between vascular risk factors and cognition at age 75 . Acta Neurol Scand 2006; 114:84-90.

39. Chui H, Zhang Q. Evaluation of dementia: a systematic study of the usefulness of the American Academy of Neurology's practice parameters. Neurology 1997; 49:925-35.

Recebido em 26/Fev/2008

Versão final reapresentada em $07 / \mathrm{Jul} / 2008$ Aprovado em 01/Out/2008 